Hydraulic Engineering Repository

Ein Service der Bundesanstalt für Wasserbau

Bollaert, Erik F. R.

Physics of Rock Scour: the Power of the Bubble

Verfügbar unter / Available at:

https://hdl.handle.net/20.500.11970/100255

Vorgeschlagene Zitierweise / Suggested citation:

Bollaert, Erik F. R. (2010): Physics of Rock Scour: the Power of the Bubble. In: Burns, Susan E.; Bhatia, Shobha K.; Avila, Catherine M. C.; Hunt, Beatrice E. (H..): Proceedings 5th International Conference on Scour and Erosion (ICSE-5), November 7-10, 2010, San Francisco, USA. Reston, Va.: American Society of Civil Engineers. S. 21-40. 


\title{
Physics of Rock Scour: the Power of the Bubble
}

\author{
E.F.R. Bollaert ${ }^{1}$, M. ASCE
}

1 President, AquaVision Engineering, Chemin des Champs-Courbes 1, CH-1024 Ecublens, Switzerland, PH $+41(0) 797751761$, email: erik.bollaertaaquavisioneng.ch

\begin{abstract}
Scour of rock downstream of high-head hydraulic structures is governed by multiphase physics of turbulent air-water mixtures impacting and eroding fractured rock. The present paper provides first of all an overview of the main physical processes and focuses on relevant break-up mechanisms of rock. Particular emphasis is given to the influence of air bubbles present in the water on scour formation. Also, relevant scaling issues are pointed out for each of the processes.

Second, based on these processes, a physics-based near-prototype scaled engineering model for scour predictions, the Comprehensive Scour Model (CSM), is being presented, as well as feedback on applications of the model to case studies and real-life projects. The CSM has been initiated in 2001 and since then been further developed and completed by applying it to real-life rock scour problems at high-head dams worldwide.

All in all, it appears that the gas phase significantly influences all stages of scour development, from the issuance of the flow at the dam crest to the formation of the scour hole downstream. The power of the air bubble reveals to be beyond our expectations.
\end{abstract}

\section{PHYSICS OF ROCK SCOUR}

Fluvial erosion of rock as it appears in the vicinity of engineering structures generally occurs following a sequence of physical-mechanical processes as illustrated at Figure 1. This figure distinguishes between the fall of an aerated water jet, the impact and diffusion of the jet through the plunge pool, the generation of dynamic pressure fluctuations at the water-rock interface, and finally instantaneous (dynamic block ejection, sudden joint break-up) and time-dependent (abrasion, progressive joint break-up, downstream displacement) rock break-up processes. Three rock breakup processes are being described more in detail:

1. rock block removal (by pressure differences in joints or shear flow),

2. rock mass fracturing (suddenly or progressively),

3. rock block peeling off (combination of removal/fracturing).

Each of these processes has its own time-scale of occurrence, ranging from instantaneous to long term. While sudden break-up actions such as block uplift are described in literature, sound assessment of progressive break-up by fracturing and 
peeling off of blocks are still in their initial phases of development. Their relevance to scour depends on the characteristics of the turbulent flow and on the shape and the protrusion of the rock blocks. For small-sized rocky material, shear flow is generally predominant, just like for a granular riverbed. For large-sized irregular rock blocks, however, the shape, dimensions and protrusion of the blocks significantly impact the failure process.

In the following, the physics of how a rock fails are explained more in detail as well as the corresponding computational modules being part of the CSM. Emphasis is thereby given to the influence of the air bubble presence in the water.

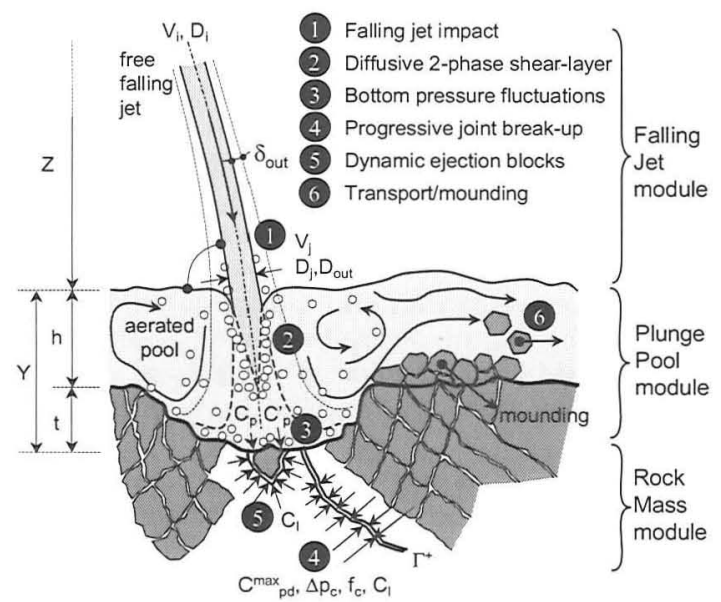

Figure 1. Physical-mechanical phenomena responsible for break-up of rock.

\section{Rock block removal}

Rock may fail by removal of distinct blocks. This may happen by uplift (quasi-vertical ejection), by horizontal displacement (shear), or by a combination of both. Beside average flow velocities and pressures near the bottom, flow turbulence is generally of importance. Which of the movements and forces are most plausible depends on the size, dimensions and protrusion of the blocks compared to the surrounding rock mass. These parameters directly define the relevance of the quasisteady and turbulent forces that may lift the block. For non-protruding blocks, only turbulent forces enhance block uplift. For highly protruding blocks, flow deviating quasi-steady forces are predominant. The Dynamic Uplift (DI) module of the CSM allows computation of uplift heights of distinct rock blocks.

\section{Rock mass fracturing}

Rock may also fail by sudden or progressive hydraulic fracturing, which is mathematically described by the theory of linear elastic fracture mechanics. Brittle fracture occurs when the stress intensity at the edges of closed-end fractures is greater 
than the in-situ fracture toughness of the rock (Bollaert, 2002). The stresses induced by water pressures are governed by the geometry of the fracture and the support of the surrounding rock. The in-situ fracture toughness of the rock depends on the type of rock, its unconfined compressive strength (UCS) and the in-situ stress field.

Second, progressive fracturing of rock occurs when the stress intensities do not exceed the fracture toughness. Prototype-scaled laboratory tests have shown the presence of air-water transient waves inside rock joints (Bollaert, 2002; Bollaert \& Schleiss, 2005). These generate cyclic pressures that, on the medium or long term, may propagate an existing fracture by fatigue, depending on the number and the intensity of pressure pulses. This failure type is time-dependent and takes an end when fracture formation is completed. The Comprehensive Fracture Mechanics (CFM) module of the CSM computes both brittle and fatigue fracturing as a function of duration of flooding.
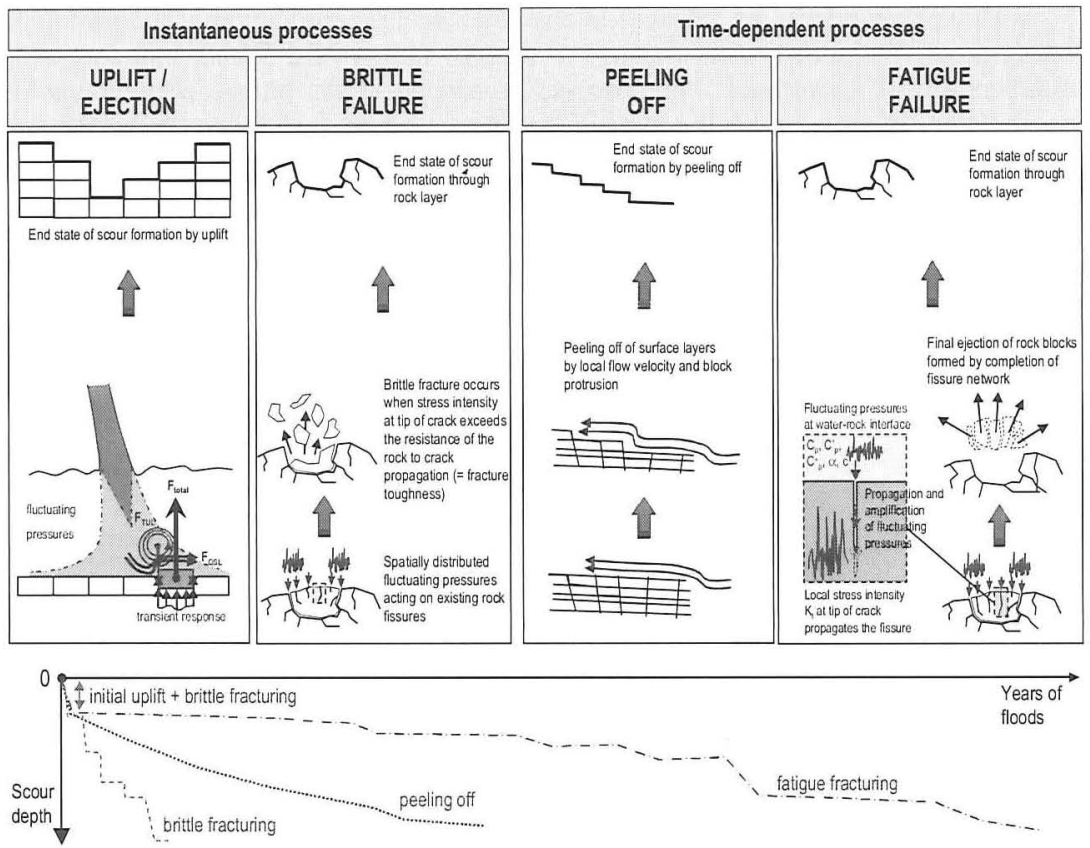

Figure 2. Principle failure mechanisms of fractured rock at hydraulic structures

\section{Rock block peeling off}

Peeling off of blocks is a specific combination of both quasi-steady pressure forces and brittle or fatigue fracturing. The phenomenon typically occurs in layered rock. The destabilizing forces are not only due to flow turbulence, but are also 
generated by a strong local flow deviation due to protrusion of the block. This flow deviation generates drag and lift forces on the exposed faces of the block, which are governed by the relative importance of the protrusion of the block and by the local quasi-steady flow velocity in its immediate proximity.

The corresponding pressures may develop brittle or fatigue fracturing of the joint between the block and the underlying rock. In case the exposed block is detached or almost detached, no further fracturing is needed to uplift the block by pressure fluctuations entering laterally into the joint. The Quasi-Steady Impulsion (QSI) module of the CSM computes peeling off of distinct rock block layers, which is particularly relevant in the case of regressive scour towards the toe of the dam of appurtenant structure.

\section{THE POWER OF THE BUBBLE}

For each of the described processes, several phases and forces work together. As such, scaling effects are inherent to any small-scale reproduction of the prototype behavior of the process in question. For aerated falling jets, Weber and Reynolds numbers are of importance. For processes occurring in the plunge pool, Reynolds numbers are often very small and air entrainment and air transfer to the bottom are both incorrect. Inside rock joints, different geometrical scales as well as different airwater wave celerities are source of discrepancies. As such, air bubbles are at the base of most of the scaling issues in rock scour because unfortunately present in all of the aforementioned physical processes. This is illustrated in Figure 3.

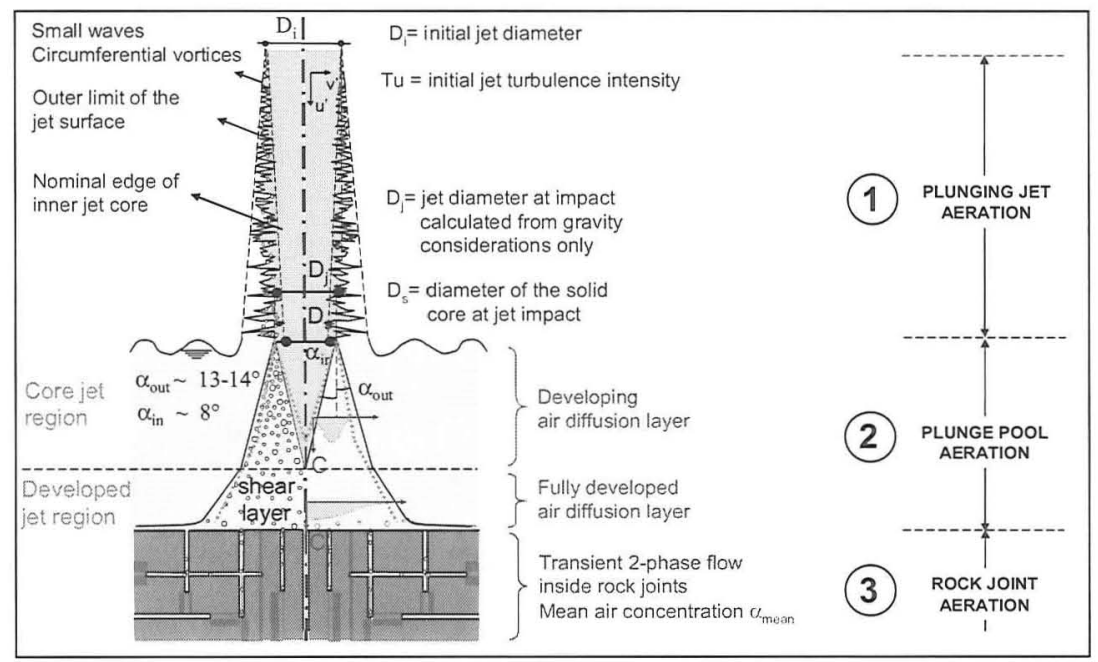

Figure 3. Air entrainment of jet, pool and rock mass. 


\section{Jet issuance from the dam}

Jet issuance conditions reproduced on a laboratory (small) scale are often affected by the following scaling effects:

1. initial jet turbulence intensity and thus air entrainment is too low

2. jet deflection angle (lip) is different than the prototype deflection angle

3. approach flow conditions in the upstream reservoir are not representative

Relevant initial jet turbulence intensities for prototype jets are between 3 and $8 \%$ (Ervine \& Falvey, 1987). The turbulence intensity is directly responsible for jet aeration and jet spread during its fall. A more detailed discussion of jet turbulence as a function of type of issuance conditions can be found in Manso et al. (2006).

\section{Jet fall through the air}

During its fall, a scaled jet exhibits the following effects:

1. inner and outer spread angles are too low

2. jet air entrainment is too low

3. shape of the jet does not deform as on prototype

4. jet trajectory does not account for air drag and wind effects

Air drag during fall is generally accounted for by means of a trajectory reduction. Air entrainment is impossible to correctly reproduce on a scale model. Shape deformation of jets during fall is very difficult to correctly reproduce at small scales.

\section{Jet diffusion through the water cushion}

When diffusing through the water cushion of the downstream plunge pool or stilling basin, the following scaling effects occur:

1. plunge pool quantity (mass) of air and volume of air at impact are low because jet velocity at impact is low

2. plunge pool water level is too stable and does not fully account for local recirculation patterns and instabilities that might be present on the prototype

3. plunge pool quantity of air (mass) at the bottom is too low

4. plunge pool volume of air at the bottom is generally too high because of the wrong quantity of air in the pool and of the lack of stagnation pressures.

Aeration aspects in pools are very complex and have been extensively studied at prototype scale by Bollaert et al. (2009). Due to stagnation, prototype water cushions exhibit a strong pressure gradient near the bottom, reducing the air volume based on the ideal gas law, whereas scaled cushions are not able to reproduce this gradient. As such, despite the low aeration at impact (due to scaled jet velocities), air volume at the bottom is generally overestimated on scale models. As such, the corresponding mean dynamic pressures are underestimated near the bottom.

Air concentrations (void fractions) were measured by means of a double fibreoptical probe on a near-prototype scaled facility. Three measurement points (MP) were selected inside the pool (Figure 4): 1) in the impingement zone of the jet (MP1), 2 ) in the transition to the wall jet region (MP2) and 3) just above the impinging jet region (MP3), $10 \mathrm{~cm}$ above the pool floor for different pool depths. 

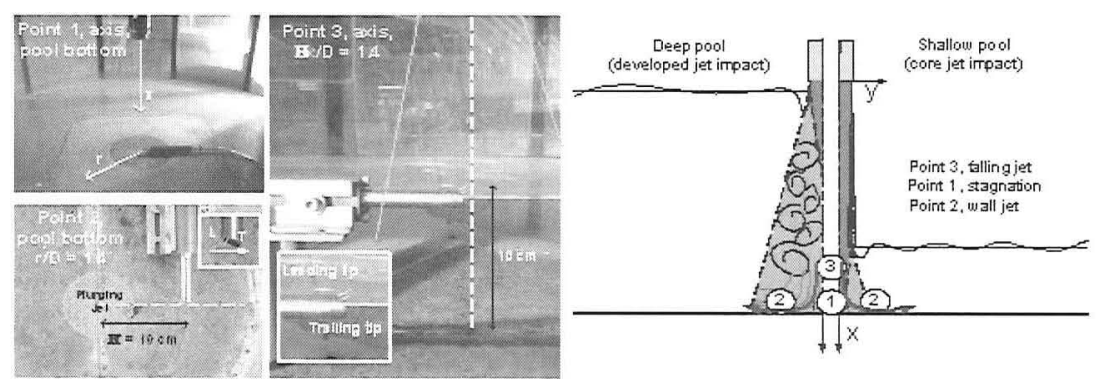

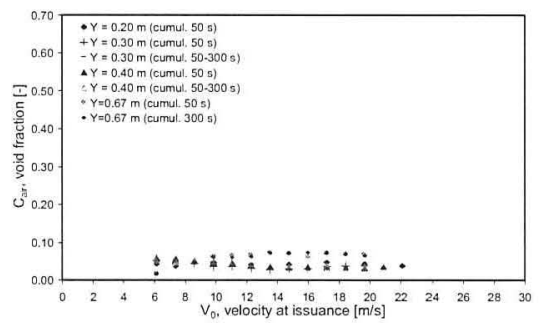

a)

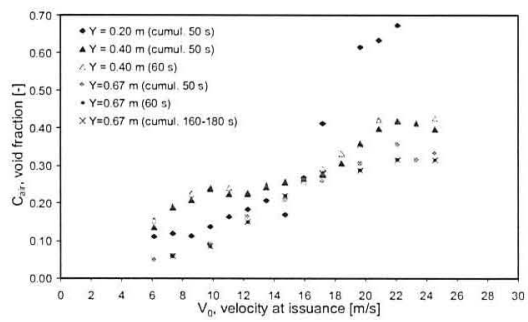

b)

Figure 4. Upper part: Positioning of optical probe and measurement points of void fraction. Lower part: Void fractions measured for different pool depths: a) at point 1 (MP1) located at the jet stagnation point (centre); b) at point 2 (MP2), in the wall jet region away from the stagnation point.

The results are presented as a function of jet issuance velocity for Y/D between 2.8 and 9.3, in which $\mathrm{Y}$ stands for the plunge pool depth and D for the jet diameter at impact. At the jet's stagnation point, measured void fractions were between 2 and $8 \%$, regardless of the jet velocity. Radially away from the stagnation point, but still along the pool floor, void fractions highly depended on the jets' issuance velocity and reached up to $40 \%$.

In other terms, at low jet velocities ( $<<10 \mathrm{~m} / \mathrm{s}$ ), void fractions at the jet's stagnation point are quite similar to the ones measured radially outwards, while at high jet velocities, $(\mathrm{V}>20 \mathrm{~m} / \mathrm{s})$, void fractions at the jet's stagnation point are $5-6$ times less than the ones measured radially outwards. A similar trend has been observed at measurement point 3 .

Void fractions are directly related to the pressure built-up when approaching the jet's stagnation point and to the sudden pressure decrease following radial jet deflection after pool floor impact. By applying the ideal gas law, the volume reduction $\Delta \mathrm{V}$ of a given quantity (mass) of air is inversely proportional to the rise in absolute pressure $\Delta \mathrm{p}$. The amount of air does not change, only the size of the bubbles changes due to a variation of absolute water pressure.

The air content at the water-rock interface influences the pressures inside the rock joints. Once the air bubbles transferred inside the joint, pressure fluctuations 
increase or decrease the corresponding volume of the mass of bubbles in a cyclic manner. Also, the mass of free air may change according to Henry's law. The presence of air inside the joint significantly modifies the compressibility of the airwater mixture and thus its ability to generate oscillations and resonance waves inside the joints (see further). As such, air bubbles are at the base of the hydraulic jacking power of a high-velocity jet. More details can be found in Bollaert $(2002,2004)$.

\section{Bottom pressure fluctuations}

Due to the significantly different turbulence and air entrainment characteristics of a small-scale pool or stilling basin, the related turbulent pressure fluctuations at the interface are also not fully representative (Bollaert et al., 2002):

1. maximum and minimum extreme pressures are too low

2. RMS (root-mean-square) values are too low

3. very high and very low frequencies are not present at small scales

4. spatial distribution of pressure fluctuations is too centralized

Hence, prototype pressures at the pool bottom are different from pressures measured on scale model facilities. Both the root-mean-square values and the extreme values are significantly higher on prototype, and the corresponding frequency spectra have considerable energy even at high frequencies. Moreover, the zone at the pool bottom that is influenced by the turbulent shear layer of the impacting jet is not well defined in reality and can extend over a wide area due to aeration and lateral displacements of the point of impact of the jet in the pool.

\section{Rock joint pressure fluctuations}

Pressures travel through joints as two-phase transient waves, whereby the jet acts as an exciter and the joint as a resonance chamber. Oscillations and extreme values are strongly depending on the air content inside the joint, as well as on the geometry (length, shape) of the joint. Small scale models are unable to correctly account for these effects. Air influence on net uplift pressures is shown in Figure 7.

Near-prototype scaled laboratory tests have shown that pressure pulses inside artificially generated rock joints may exhibit amplifications of several times the corresponding pressure pulse at the water-rock interface (entrance of the joint). This clearly points out the importance of the air presence and is illustrated at Figure 5.
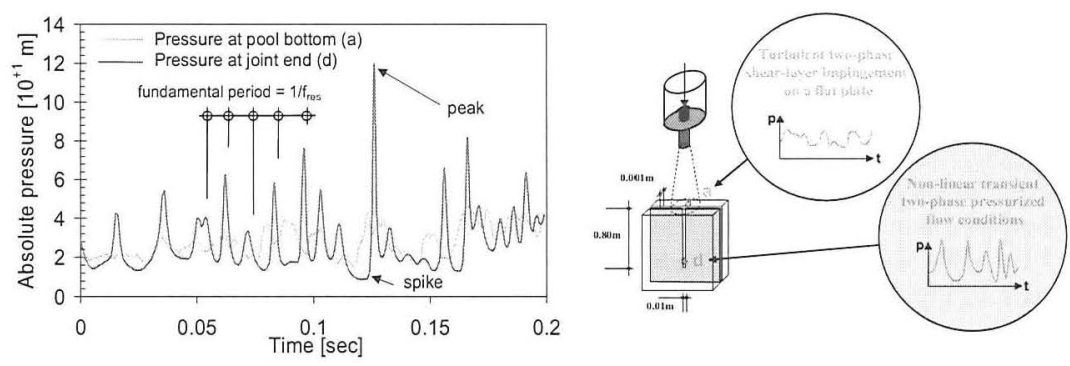


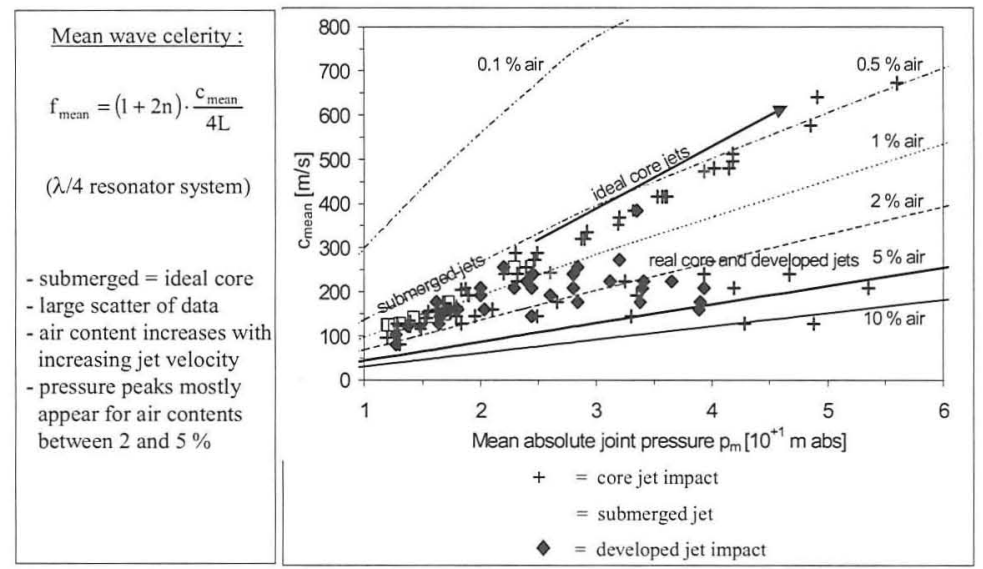

Figure 5. Air concentrations and mean wave celerities inside rock joints impacted by aerated and non-aerated (submerged) high-velocity jets.

\section{Rock mass resistance to scour}

Small-scale physical models often make use of binders such as cement, clay, etc. to simulate the resistance of partially fractured rock against scour. This, however, has proven to be unreliable and unable to accurately model both the real shape and the extent of a plunge pool scour hole.

It is evident that mixtures of sand/gravel and binders cannot replace the much more complex prototype behavior of rock when it comes to fracturing processes and dynamic ejection of rock blocks. The former process is governed by the fracture toughness of the rock mass and by the real geometry of the joints. The latter process depends on dynamic pressure pulses over and under the blocks, which are directly related to local turbulent conditions near the block in question.

Typical shortcomings of scale models are the lack of steep slopes of the modeled scour hole, and the appearance of a downstream mound that is way too important. Unfortunately, both aspects have significant influence on scour formation.

\section{THE COMPREHENSIVE SCOUR MODEL (CSM)}

A physics based scour prediction model, the Comprehensive Scour Model (Bollaert, 2002, 2004; Bollaert \& Schleiss, 2005), has been developed based on the aforementioned processes. It is called comprehensive in the sense that it incorporates the major physics relevant to scour in an easily understandable manner, i.e. by using mathematics of the physical laws that are both representative for the phenomena in question but at the same time easy to understand.

The model is applicable to any kind of brittle fractured medium, i.e. fractured rock, strong clays, concrete, etc. Typical fields of application are spillways and stilling basins, bridge piers, concrete fracturing of spillway chutes, uplift of stilling basin concrete linings, uplift of anchored sidewalls and protection slabs, a.s.o. 
It uses linear elastic fracture mechanics to express hydraulic jacking in the fractured medium of interest. Second, dynamic uplift of blocks of the fractured medium due to net uplift forces and impulsions is being simulated. The hydraulic action for each failure mechanism is determined along the scour critical parts of the liquid-solid interface. The scour resistance of the fractured medium is expressed by its main geomechanical characteristics. Interaction between the progressing scour hole and its influence on the hydraulic action is being accounted for.

The model computes failure of fractured rock by fracturing, uplift or peeling off. The structure consists of 3 modules: the falling jet, the plunge pool and the rock mass. The latter directly implements the different failure mechanisms.

\section{Falling Jet Module}

This module describes how the hydraulic and geometric characteristics of the jet are transformed from dam issuance down to the plunge pool (Figure 1). Three main parameters characterize the jet at issuance: the velocity $\mathrm{V}_{\mathrm{i}}$, the diameter (or width) $D_{i}$ and the initial turbulence intensity $T u$, defined as the ratio of velocity fluctuations to the mean velocity. The jet trajectory is based on ballistics and air drag. The jet module computes the longitudinal location of impact, the total trajectory length $L$ and the velocity and diameter at impact $V_{j}$ and $D_{j}$.

\section{Plunge Pool Module}

This module describes the characteristics of the jet when traversing the plunge pool and defines the water pressures at the water-rock interface. The plunge pool water depth $Y$ and bottom shape are essential. The water depth $Y$ and jet diameter at impact $D_{j}$ determine the ratio $Y / D_{j}$, which is directly related to jet diffusion. The most relevant pressures are the mean dynamic pressure coefficient $\mathrm{C}_{\mathrm{pa}}$ and the root-meansquare (rms) coefficient of the fluctuating dynamic pressures $\mathrm{C}_{\mathrm{pa}}^{\prime}$, both measured directly under the centerline of the jet. These coefficients are influenced by the degree of confinement of the pool bottom, generating upward oriented return currents that enhance energy dissipation inside the pool.

\section{Rock Mass Module}

The pressures at the bottom are used for determination of pressures inside rock joints. The main parameters are: the maximum dynamic pressure coefficient $C^{\max }{ }_{p}$, the characteristic amplitude $\Delta p_{c}$ and frequency $f_{c}$ of pressure cycles and the maximum dynamic impulsion coefficient $C^{\max }{ }_{1}$. The first parameter is relevant to brittle propagation of closed-end rock joints. The second and third parameters express timedependent propagation of closed-end rock joints. The fourth parameter is used to define dynamic uplift of rock blocks formed by open-end rock joints.

The maximum dynamic pressure $\mathrm{C}_{\mathrm{p}}^{\max }$ is obtained through multiplication of the rms pressure $C_{p a}^{\prime}$ with an amplification factor $\mathrm{F}^{+}$, and by superposition with the mean dynamic pressure $\mathrm{C}_{\mathrm{pa}}$. The amplification depends on the air content and the product of $\mathrm{C}_{\mathrm{pa}}^{\prime}$ times $\mathrm{F}^{+}$results in a maximum pressure, written as (Bollaert, 2002 \& 2004): 


$$
\mathrm{P}_{\max }[\mathrm{Pa}]=\gamma \cdot \mathrm{C}_{\mathrm{p}}^{\max } \cdot \frac{\mathrm{V}_{\mathrm{j}}^{2}}{2 \mathrm{~g}}=\gamma \cdot\left(\mathrm{C}_{\mathrm{pa}}+\Gamma^{+} \cdot \mathrm{C}_{\mathrm{pa}}^{\prime}\right) \cdot \frac{\mathrm{V}_{\mathrm{j}}^{2}}{2 \mathrm{~g}}
$$

The characteristic amplitude of the pressure cycles, $\Delta \mathrm{p}_{\mathrm{c}}$, is determined by the maximum and minimum pressures of the cycles. The characteristic frequency of pressure cycles $f_{c}$ follows the assumption of a perfect resonator system (see Figure 5) and depends on the air concentration in the joint $\alpha_{i}$ and on the length of the joint $\mathrm{L}_{\mathrm{f}}$.

\section{Comprehensive Fracture Mechanics (CFM)}

The resistance of the rock against fracture propagation has to be determined. The cyclic character of pressures in joints makes it possible to describe joint propagation by fatigue stresses occurring at their tip. This can be described by Linear Elastic Fracture Mechanics. Joint propagation distinguishes between brittle and timedependent propagation. The former happens for a stress intensity equal to or higher than the fracture toughness of the rock. The latter is occurring in the opposite case. Joints may then be propagated by fatigue. Failure by fatigue depends on the frequency and the amplitude of the load cycles. Stresses are characterized as follows:

$$
\mathrm{K}_{1}=\mathrm{P}_{\max } \cdot \mathrm{F} \cdot \sqrt{\pi \cdot \mathrm{L}_{\mathrm{f}}}
$$

in which $\mathrm{K}_{\mathrm{I}}$ is in $\mathrm{MPa} \sqrt{\mathrm{m}}$ and $\mathrm{P}_{\max }$ in $\mathrm{MPa}$. The boundary correction factor $\mathrm{F}$ depends on the type of crack and on its persistency, defined as a/B or b/W in Figure 6.
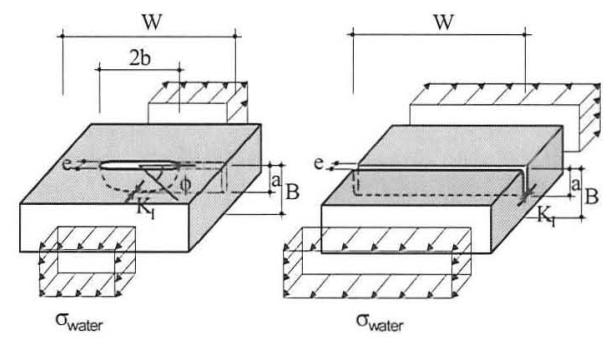

Figure 6. Types of rock joint configurations modelled in the CSM.

This figure presents two basic configurations for partially jointed rock. The choice of the most relevant geometry depends on the type and the degree of jointing of the rock. The first crack is of semi-elliptical shape and partially sustained by the surrounding rock mass in two horizontal directions. Corresponding stress intensity factors should be used in case of low to moderately jointed rock. The second crack is single-edge notched and of two-dimensional nature. Support from the surrounding rock mass is only exerted perpendicular to the plane of the notch and, as a result, stress intensity factors will be substantially higher. Thus, it is appropriate for significantly to highly jointed rock. For practice, $\mathrm{F}$ values of 0.5 or higher are considered to correspond to completely broken-up rock, i.e. dynamic uplift becomes more relevant than fracturing. For values of 0.1 or less, a tensile strength approach is more plausible. 
However, most of the values in practice can be considered between 0.20 and 0.40 , depending on the type and number of joint sets, the degree of weathering, joint interdistances, etc. The fracture toughness $\mathrm{K}_{\mathrm{Ic}}$ has been related to the mineralogical type of rock and to the unconfined compressive strength UCS. Furthermore, corrections are made to account for the loading rate and the in-situ stress field. Hence, the in-situ fracture toughness $\mathrm{K}_{\mathrm{I}, \mathrm{ins}}$ is based on literature data and written as:

$$
\mathrm{K}_{\mathrm{I} \text { ins, UCS }}=(0.008-0.010) \cdot \mathrm{UCS}+\left(0.054 \cdot \sigma_{\mathrm{c}}\right)+0.42
$$

in which $\sigma_{\mathrm{c}}$ represents the confinement horizontal in-situ stress and T, UCS and $\sigma_{\mathrm{c}}$ are in MPa. Instantaneous joint propagation will occur if $\mathrm{K}_{1} \geq \mathrm{K}_{\mathrm{I}, \mathrm{ins}}$. If this is not the case, joint propagation is expressed as follows:

$$
\frac{\mathrm{dL}_{\mathrm{f}}}{\mathrm{dN}}=\mathrm{C}_{\mathrm{r}} \cdot\left(\Delta \mathrm{K}_{1} / \mathrm{K}_{\mathrm{lc}}\right)^{\mathrm{m}_{\mathrm{r}}}
$$

in which $\mathrm{N}$ is the number of pressure cycles. $\mathrm{C}_{\mathrm{r}}$ and $\mathrm{m}_{\mathrm{r}}$ are material parameters that are determined by fatigue tests and $\Delta \mathrm{K}_{\mathrm{I}}$ is the difference of maximum and minimum stress intensity factors. To implement time-dependent joint propagation into the model, $m_{r}$ and $C_{r}$ have to be known. A calibration for granite (Cahora-Bassa Dam; Bollaert, 2002) resulted in $\mathrm{C}_{\mathrm{r}}=1 \mathrm{E}-8$ for $\mathrm{m}_{\mathrm{r}}=10$.

\section{Dynamic Impulsion (DI)}

The last hydrodynamic parameter of importance is the maximum dynamic impulsion $\mathrm{C}^{\mathrm{max}}{ }_{\mathrm{I}}$ in an open-end joint (underneath single block), obtained by time integration of net forces on the block (pressures under and over block, immerged weight of block and eventually shear and interlocking forces).

$$
I=\int_{0}^{\Delta t p u l s e}\left(F_{u}-F_{o}-G_{b}-F_{s h}\right) \cdot d t=m \cdot V_{\Delta t p u l s e}
$$

in which $F_{u}$ and $F_{o}$ are the forces under and over the block, $G_{b}$ is the submerged weight of the block and $F_{\text {sh }}$ represents the shear and interlocking forces. The shape of a block and the type of rock define the immerged weight. Shear and interlocking forces depend on the joint pattern and the in-situ stresses. As a first approach, they can be neglected. The pressure field over the block is governed by jet diffusion. The pressure field under the block corresponds to transient pressure waves.

Uplift of a block may be computed by defining at each time instant the uplift forces on the block, together with the resistant forces defined by its mass and by eventual shear and interlocking forces between the block and the surrounding rock. The force balance has to be established following the potential orientation of movement.

The first step is to define the maximum net impulsion $I_{\max } . I_{\max }$ is defined as the product of a net force and a time period. The corresponding pressure is made nondimensional by the jet's kinetic energy $\mathrm{V}^{2} / 2 \mathrm{~g}$. This results in a net uplift pressure coefficient $\mathrm{C}_{\mathrm{up}}$. The influence of air presence on this coefficient is shown in Figure 7 (left side). The time period is made non-dimensional by the travel period that is characteristic for pressure waves inside rock joints, i.e. $\mathrm{T}=2 \cdot \mathrm{L}_{\mathrm{f}} / \mathrm{c}$. This results in a time coefficient $\mathrm{T}_{\text {up }}$. Hence, the non-dimensional impulsion coefficient $\mathrm{CI}$ is defined 
by the product $\mathrm{C}_{\text {up }} \cdot \mathrm{T}_{\text {up }}=\mathrm{V}^{2} \cdot \mathrm{L} / \mathrm{g} \cdot \mathrm{c}[\mathrm{m} \cdot \mathrm{s}]$. The maximum net impulsion $\mathrm{I}_{\max }$ is obtained by multiplication of $\mathrm{C}_{\mathrm{I}}$ by $\mathrm{V}^{2} \cdot \mathrm{L} / \mathrm{g} \cdot \mathrm{c}$. Prototype-scaled analysis of uplift pressures resulted in the following expression for $\mathrm{C}_{\mathrm{I}}$ :

$$
C_{I}=0.0035 \cdot\left(\frac{Y}{D_{j}}\right)^{2}-0.119 \cdot\left(\frac{Y}{D_{j}}\right)+1.22
$$

Failure of a block is expressed by the displacement it undergoes due to the net

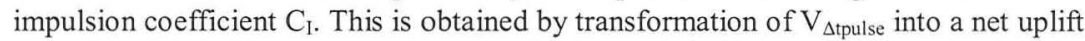
displacement $h_{\text {up }}$. The net uplift displacement that is necessary to eject a rock block from its matrix is difficult to define. It depends on the protrusion and the degree of interlocking of the blocks. A calibration on Cahora-Bassa Dam (Bollaert, 2002) resulted in a critical net uplift displacement of 0.20 .

Nevertheless, in reality, block movement and uplift forces are highly correlated. Experimental research is actually ongoing at the Swiss Federal Institute of Technology in Lausanne to solve this complex correlation (Federspiel et al., 2009). An artificial rock block has been equipped with pressure and acceleration sensors to detect the direct relation between the pressures over and under the block and its detailed movements. The block is being impacted by a near-prototype air-water jet.

\section{Quasi-Steady Impulsion (QSI)}

Peeling off of rock blocks is a specific combination of both quasi-steady forces and brittle or fatigue fracturing. The phenomenon typically occurs in layered rock, such as sedimentary rock. If is often responsible for regressive erosion towards the toe of the dam. The destabilizing forces are not due to flow turbulence alone, but are principally generated by the flow deviation due to a protrusion "e" of the block along the bottom (e $e_{\text {block }}$ in Figure 7 , right side). This flow deviation generates drag and lift forces on the exposed faces of the block, which are governed by the relative importance of the protrusion of the block into the flow and by the local quasi-steady flow velocity in the immediate proximity of the block ( $\mathrm{V}_{\text {backflow }}$ in Figure 7 ).

These forces may develop brittle or fatigue fracturing of the joint between the block and the underlying rock mass. In many cases, the exposed block is detached or almost detached and no further fracturing is needed to uplift the block from its mass.
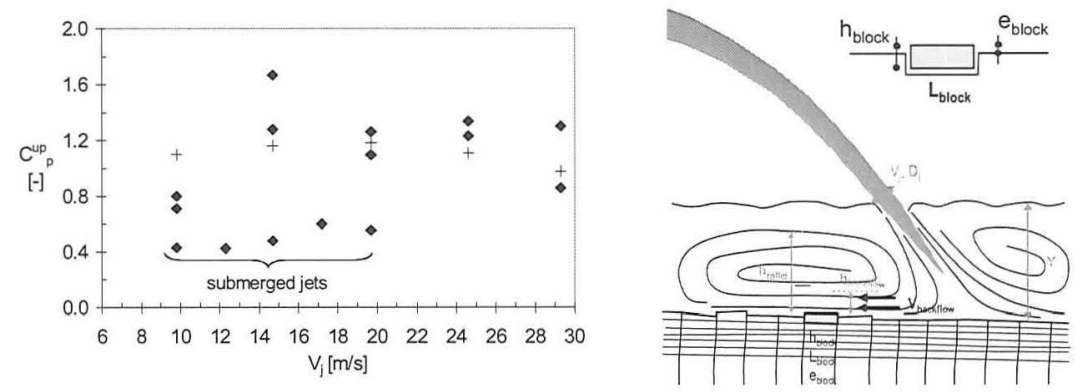

Figure 7. left: $\mathrm{C}_{\text {up }}$ for aerated and non-aerated jets; right: Peeling off of rock blocks at surface during flow event. 


\section{APPLICATION TO KARIBA DAM (ZAMBIA-ZIMBABWE)}

Kariba Dam is a $128 \mathrm{~m}$ high concrete arch dam on the Zambezi River, situated on the border between Zambia and Zimbabwe. Since 1962, spillway discharges from Kariba Dam have eroded an important scour hole into the gneiss rock, which extends since 1982 about $80 \mathrm{~m}$ below the initial river bed (Mason \& Arumugam, 1985).

Use of estimated annual flood periods and in-situ measured scour formation allowed calibrating the CSM model to predict future scour formation as a function of time (Bollaert, 2005). Especially the time-related parameters of the CSM model have been adapted to the long-duration observed prototype scour (20 years of scour followup between 1962 and 1981).

After dam construction in 1959, a large scour hole quickly formed in the downstream fractured rock. Typical spillway discharges and average tailwater levels are available, and the average time duration of floods has been estimated at about 3 months. Furthermore, after each major flood period between 1962 and 1981, a detailed bathymetric survey of the scour hole has been carried out. Results of these surveys can be found in Mason \& Arumugam (1985).

The spillway consists of 6 rectangular gate openings of roughly $8.8 \mathrm{~m}$ by 9.1 $\mathrm{m}$, for a total discharge of about $9^{\prime} 500 \mathrm{~m}^{3} / \mathrm{s}$. The gate lips are situated at $456.5 \mathrm{~m}$ a.s.l. The minimum and maximum reservoir operating levels are 475.5 and $487.5 \mathrm{~m}$ a.s.1. The downstream tailwater level is situated between 390 and $410 \mathrm{~m}$ a.s.l., depending on the number of gates functioning. An average value of $400 \mathrm{~m}$ a.s.l. has been assumed for the computations. The net head difference results in typical jet outlet velocities of $21.5 \mathrm{~m} / \mathrm{s}$.

Scour formation in the rock mass reached a level of $306 \mathrm{~m}$ a.s.l. in 1981, i.e. about $80 \mathrm{~m}$ down the initial bedrock level. The rock mass is sound gneiss with a degree of fracturing that is not known precisely. Without further noticeable information on the rock mass quality, the computations have been performed for a set of conservative, average and beneficial parametric assumptions. Parametric analysis points out the influence of this uncertainty on the computed scour formation.

The spillway discharges are generally performed for varying gate numbers, gate openings and operations, as a function of already formed scour. This results in complex and varying hydraulics. In the following, a 2D simplified approach is considered, assuming only one jet and a (considered reasonable) average gate opening of $75 \%$. The time durations of the floods also vary from year to year. Nevertheless, it is considered that the flood season generally takes several months in this region. Hence, an average duration of 3 months or 90 days per year has been assumed for the scour computations.

Table 1 summarizes the parametric assumptions made for the rock properties. The main scour influencing parameters are the UCS (Unconfined Compressive Strength) strength, the initial degree of fracturing and the form of the joints.

Scour evolution with time is presented in Figure 8 for a range of different UCS strengths. Significant differences in scour formation are observed, underlying the need for sound UCS knowledge. Especially at lower UCS strengths, scour formation becomes sensitive to the absolute value. Based on the in-situ measured scour hole, the rock mass strength to be used in the calibrated CSM should be between 75 and $100 \mathrm{MPa}$. 
Table 1. Rock mass properties under different parametric assumptions

\begin{tabular}{|l|c|ccc|l|}
\hline \multicolumn{1}{|c|}{ Property } & Symbol & CONSERV & AVERAGE & BENEF & Unity \\
\hline Unconfined Compressive Strength & $\mathrm{UCS}$ & 100 & 125 & 150 & $\mathrm{MPa}$ \\
Density rock & $\gamma_{\mathrm{r}}$ & 2600 & 2700 & 2800 & $\mathrm{~kg} / \mathrm{m}^{\mathrm{s}}$ \\
Typical maximum joint length & $\mathrm{L}$ & 1 & 1 & 1 & $\mathrm{~m}$ \\
Vertical persistence of joint & $\mathrm{P}$ & 0.12 & 0.25 & 0.55 & - \\
Form of rock joint & - & single-edge & elliptical & circular & - \\
Tightness of joints & - & tight & tight & tight & - \\
\hline Total number of joint sets & $\mathrm{N}_{\mathrm{j}}$ & $3+$ & 3 & $2+$ & - \\
Typical rock block length & $\mathrm{I}_{\mathrm{b}}$ & 1 & 1 & 1 & $\mathrm{~m}$ \\
Typical rock block width & $\mathrm{b}_{\mathrm{b}}$ & 1 & 1 & 1 & $\mathrm{~m}$ \\
Typical rock block height & $\mathrm{z}_{\mathrm{b}}$ & 0.5 & 0.75 & 1 & $\mathrm{~m}$ \\
\hline Joint wave celerity & $\mathrm{C}$ & 150 & 125 & 100 & $\mathrm{~m} / \mathrm{s}$ \\
\hline
\end{tabular}

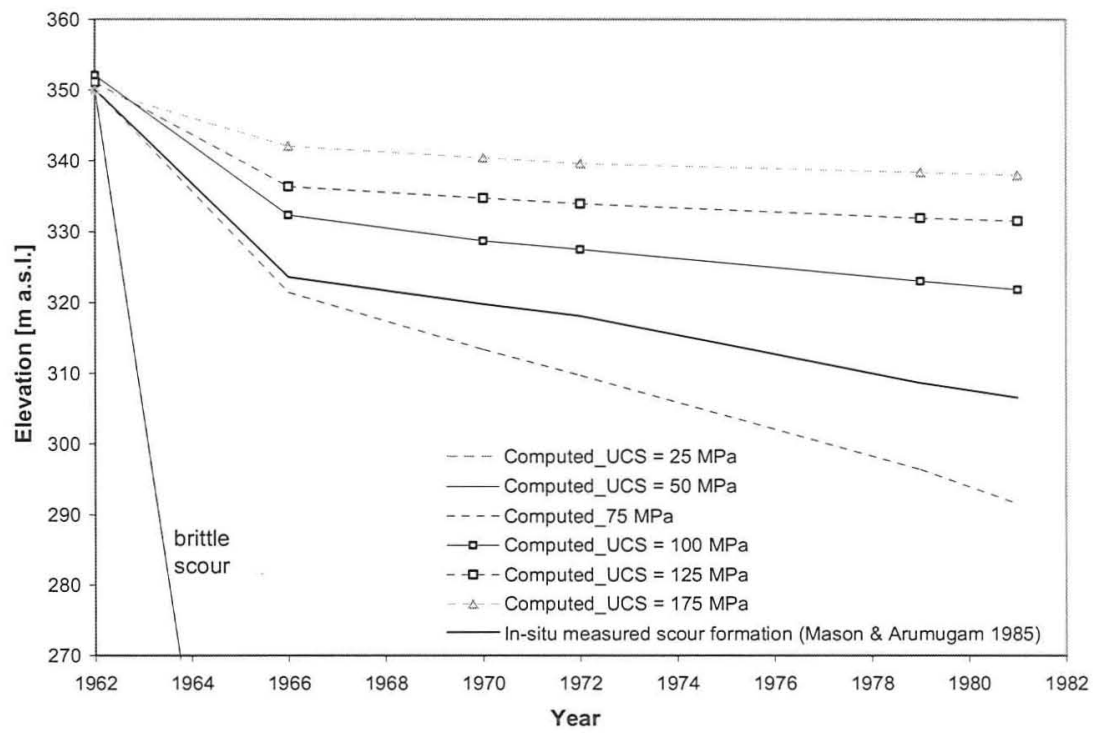

Figure 8. Prototype-scaled scour reproduction at Kariba Dam using the CSM for different UCS strengths (Bollaert, 2005) and comparison with in-situ scour hole.

\section{APPLICATION TO FOLSOM DAM (US)}

The DI and CFM modules have been applied to the lined stilling basin of Folsom Dam, a concrete gravity dam with a height of about $100 \mathrm{~m}$ situated near Sacramento, California, US. Due to a significant increase of the initial PMF estimates, the outlet works of the dam were initially proposed to be increased. This 
would have resulted in a significant increase of turbulent pressure fluctuations impacting the concrete lining of the downstream stilling basin.

Hence, at first, a concrete lining stability study has been performed, pointing out the need for significant additional steel anchors to keep the slabs in place. Following this, a rock scour study has been performed of the fractured rock mass underneath the concrete lining, to check for scour formation and extent under extreme conditions and following potential lining failure. In the following, examples are provided of results that were generated for the PMF event (Bollaert et al., 2006).

Figure 9 presents a plan and perspective view of the final 3D shape of the scour hole through the rocky foundation of the stilling basin. One can easily detect the areas of impact of the jets issuing from the outlets. The model predicts 6-9 $\mathrm{m}$ of scour formation within the first 12-24 h of a PMF flood, while subsequent scour deepening would need far more time to occur. No scour forms at the toe of the dam.

$3 \mathrm{D}$ view of dam and basin geometry

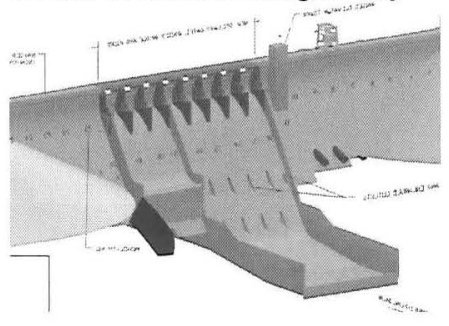

Plan view dam and basin geometry
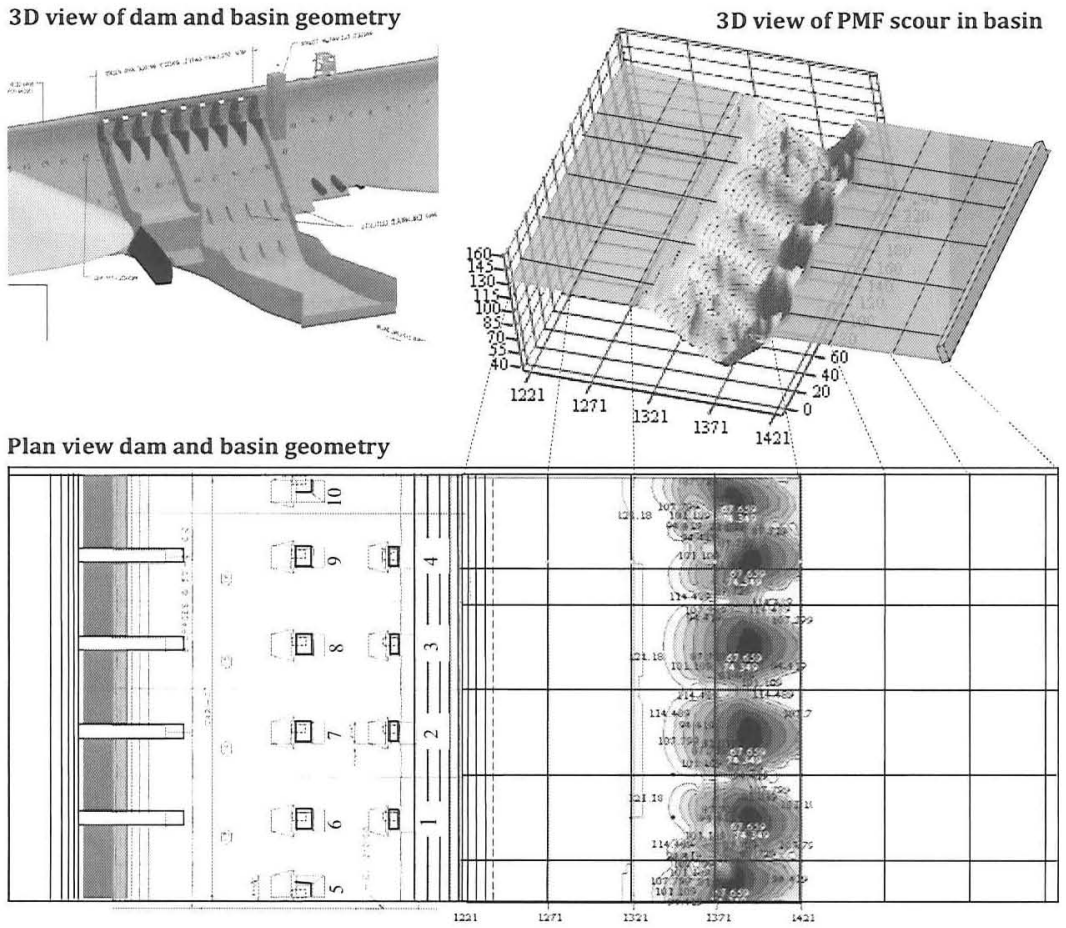

Figure 9. Plan view and perspective view of scour contours in stilling basin due to upper tiers functioning. 


\section{APPLICATION TO TUCURUI DAM (BRAZIL)}

Tucurui Dam Spillway is located on the Tocantins River in northern Brazil. The spillway is characterized by an ogee type gate-controlled structure topped by 23 radial gates ( $20.75 \mathrm{~m}$ high $\times 20 \mathrm{~m}$ wide), a compact flip bucket and a $50 \mathrm{~m}$ deep plunge pool (Figure 10). The design discharge is $110,000 \mathrm{cms}$ under a gross head of 60 to 70 $\mathrm{m}$. Hydraulics laboratory model tests resulted in the forecast of a satisfactory scouring behavior for a pre-excavated plunge pool at an elevation of $-40 \mathrm{~m}$ a.s.l.

Scour formation in the downstream plunge pool has been described by a series of bathymetric surveys since 1984 . These showed that, as predicted by the laboratory tests, the maximum observed scour depth was of only $5 \mathrm{~m}$. It was assumed that this erosion is related to removal of partially detached rock blocks during initial spillage. These blocks were fractured and detached by blasting during dam construction.
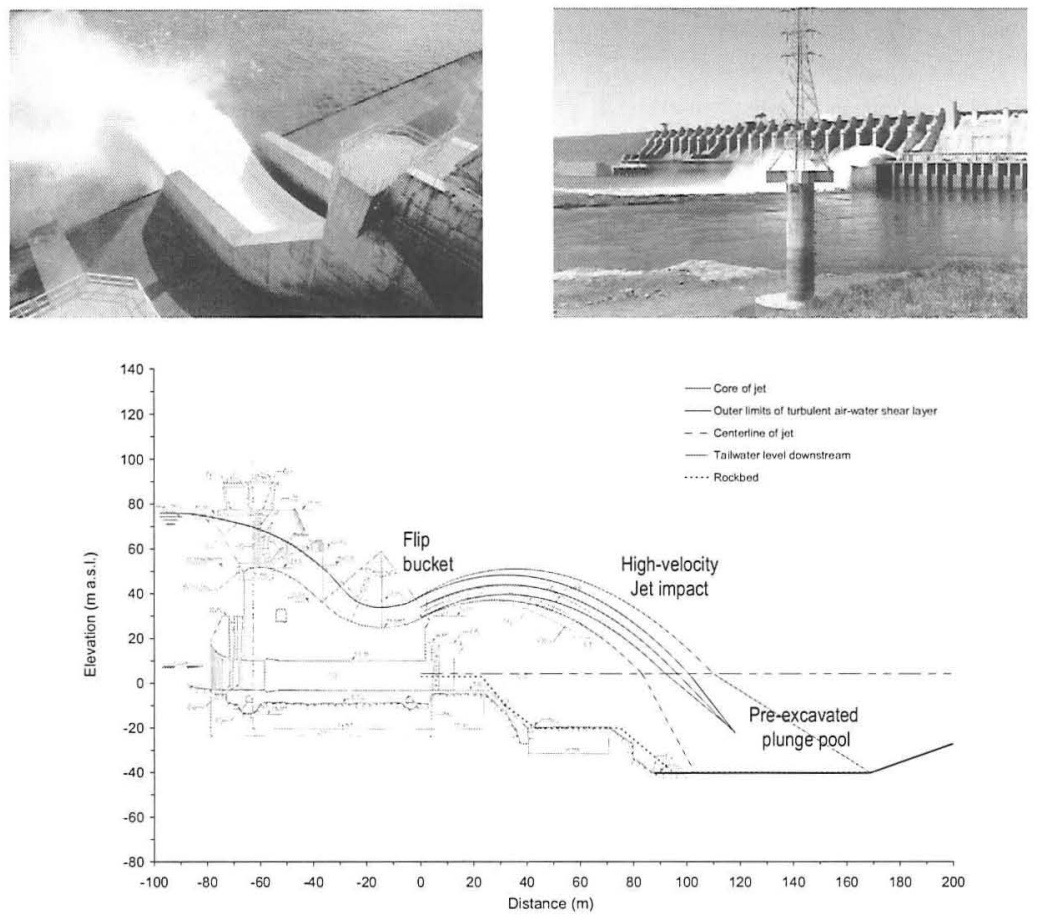

Figure 10. Photos and longitudinal section of spillway at Tucurui Dam.

Hence, it may be stated that the pre-excavated plunge pool behaves like expected during dam construction. For a recorded period of 17 years, incorporating 6 flood events of more than $31^{\prime} 000 \mathrm{~m}^{3} / \mathrm{s}$ and a maximum value of $43^{\prime} 400 \mathrm{~m}^{3} / \mathrm{s}$, no significant scour formation could be observed. 
The CSM model has first of all been calibrated based on the assumption that, for flood events of up to $50^{\prime} 000 \mathrm{~m}^{3} / \mathrm{s}$, no significant scour forms at the plunge pool bottom. Second, the model has been applied to a fictitious design flood event with a discharge of $110^{\prime} 000 \mathrm{~m}^{3} / \mathrm{s}$ (Bollaert \& Petry, 2006).

\section{Comprehensive Fracture Mechanics (CFM) results}

By using realistic parametric assumptions regarding rock resistance to scour, scour formation down to a plunge pool bottom level of about $-54.9 \mathrm{~m}(=14.9 \mathrm{~m}$ of additional scour depth) has been computed for a design flood duration of 2 months.

Second, for 8 months of design flood, the corresponding plunge pool scour elevation is at $-56.5 \mathrm{~m}(=16.5 \mathrm{~m}$ of additional scour depth). Finally, on the long term (= after 80 months of design flood), a maximum scour elevation of $-59.2 \mathrm{~m}$ has been computed (=19.2 m of additional scour depth). In other words, even during very long periods of design discharge at Tucurui Dam, potential scour formation would still remain within controllable limits.

\section{Dynamic Impulsion (DI) results}

Computed scour becomes more important than for the CFM model, with scour at $-63 \mathrm{~m}$ for beneficial rock resistance assumptions and down to $-94 \mathrm{~m}$ for conservative rock resistance assumptions. For average (most reasonable) parametric assumptions, scour goes down to $-79 \mathrm{~m}$, i.e. $39 \mathrm{~m}$ of additional scour depth.

Nevertheless, it has to be kept in mind that the DI model results largely depend on the assumed ratio of rock block height to side length. Under conservative assumptions, this ratio has been taken equal to 0.5 . Under average assumptions, this ratio has been taken equal to 0.75 . Using the DI method also means that only completely detached rock blocks would be present at depth in the plunge pool bottom, which is most probably not the case.

\section{APPLICATION TO KARAHNJUKAR DAM (ICELAND)}

Landsvirkjun, the National Power Company in Iceland, has completed in 2008 the 690 MW HEP Kárahnjúkar project in eastern Iceland. The main dam is a $200 \mathrm{~m}$ high CFRD dam. The bottom outlet of Kárahnjúkar Dam is $5.2 \mathrm{~m}$ wide, $6 \mathrm{~m}$ high and is concrete lined (Figure 11).

The first $50 \mathrm{~m}$ are near horizontal, followed by a slope change down to $5 \%$ for the remaining $300 \mathrm{~m}$. The invert and side walls are concrete lined up to a height of $3.5 \mathrm{~m}$. The tunnel ends with a double curvatured flip bucket that projects the water jet with an angle between 21 and $28^{\circ}$ into the downstream canyon.

Numerical computations have been performed of potential scour formation of the canyon following bottom outlet operation. Both downstream tailwater level and duration of discharge have been accounted for. The results show that scour formation in the canyon riverbed will remain limited (Figure 12). Scour may occur under the form of uplift and displacement of loose blocks that are already present at the riverbed. Subsequent fracturing and block formation of the in-situ rock mass will take considerable time to occur and will most probably not result in excessive scour formation. 


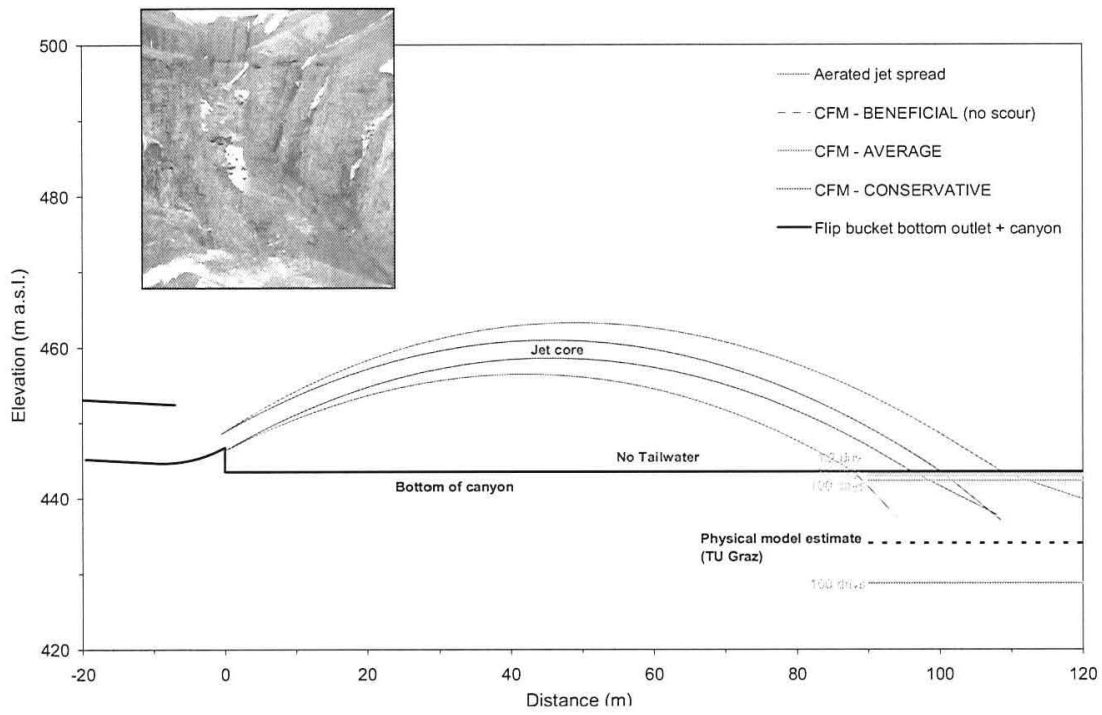

Figure 11. Scour formation in canyon as predicted by CFM module.

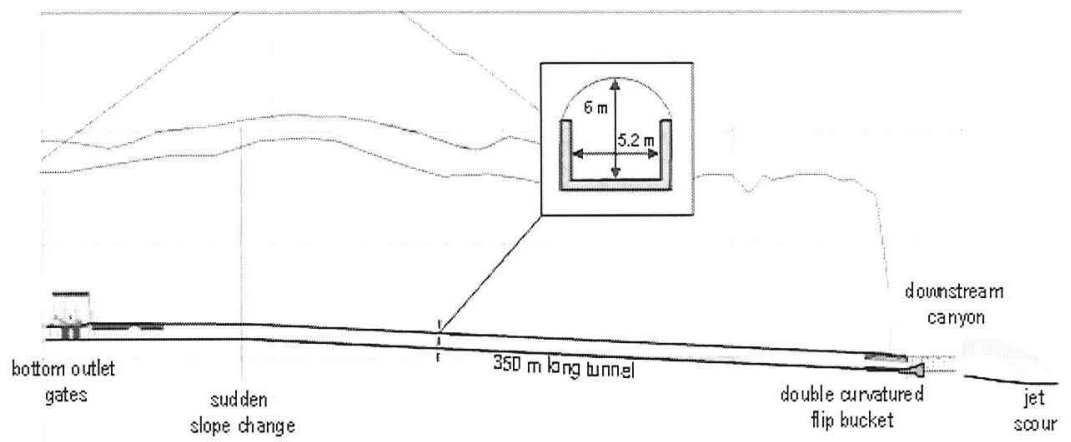

Figure 12. Longitudinal profile of bottom outlet and flip bucket. 


\section{CONCLUSIONS}

Sound assessment of the physical processes responsible for rock mass failure at high-head dams and plunge pools has shown the importance of air bubbles present in the water. The bubbles do not only influence the jet during its fall and the diffusion of the jet through the pool and the pressure fluctuations it generates, but also govern the cyclic pressure waves of the water inside the underlying rock joints. As such, the bubbles are directly relevant to hydraulic jacking of the rock mass.

Furthermore, a large series of near-prototype scaled laboratory tests have shown that use of small scale physical models may significantly alter the outcome of scour depth predictions. Especially flow turbulence and jet and pool aeration should be correctly reproduced in order to obtain sound scour predictions. The complex three-phase behavior of fractured rock impacted by turbulent pressures cannot be reproduced on a small scale model.

Hence, based on a sound analysis of the multi-phase physics of rock failure mechanisms and a large series of near-prototype scaled laboratory recordings of water pressures in artificially generated rock joints, a numerical scour prediction model has been developed. The model predicts scour formation in any type of fractured medium by computing fracture propagation, dynamic uplift and peeling off of blocks.

Appropriate calibration of the model needs the assessment of a number of hydraulic, hydrologic, geometric and geomechanic parameters. Especially the time duration and average discharge values of floods, the intrinsic rock mass strength and the initial degree of fracturing of the rock mass have to be known in a sufficiently precise manner to obtain values that can be used for practice.

When these values are available or can be reasonably estimated based on insitu observations or based on values from similar dam sites, the numerical model can be used to predict further scour formation as a function of time and/or to evaluate the ultimate scour depth on the long term.

During the last 10 years, the model has been widely used for scour prediction and/or mitigation at high-head dams and in stilling basins. Within this framework, based on historic floods and observed scour formation, the numerical model could be calibrated and thus used to predict potential future scour formation with time. Feedback from practice has shown that the model provides significant insight into the different rock failure mechanisms and is able to assist the engineer when designing scour mitigation measures.

\section{REFERENCES}

Bollaert, E.F.R. (2002). "Transient water pressures in joints and formation of rock scour due to high-velocity jet impact." PhD Thesis $N^{\circ} 2548$, Ecole Polytechnique Fédérale de Lausanne, Switzerland.

Bollaert, E.F.R. (2004). "A comprehensive model to evaluate scour formation in plunge pools." Int. Journal of Hydropower \& Dams, 2004(1), pp. 94-101.

Bollaert, E.F.R. and Schleiss, A. (2005). "Physically Based Model for Evaluation of Rock Scour due to High-Velocity Jet Impact." J. of Hydr. Eng., Vol. 131, N ${ }^{\circ}$ 3, pp. 153-165. 
Bollaert, E.F.R., Vrchoticky, B. and Falvey, H.T. (2006). "Extreme Scour Prediction at High-Head Concrete Dam and Stilling Basin (United States)." 3rd Intl. Scour and Erosion Conference, Amsterdam, 2006.

Bollaert, E.F.R. \& Petry, B. (2006). "Application of a physics based scour predictionmodel to Tucurui dam spillway (Brazil)." Intl. Symp. On Hydr. Structures, Ciudad Guyana, Venezuela.

Bollaert, E.F.R., Manso, P. and Schleiss, A.J. (2009). "Discussion of: "Effect of jet aeration on hydrodynamic forces on plunge pool floors." Canadian Journal of Civil Engineering, Vol. 36, pp. 524-526.

Council for Scientific and Industrial Research, Report NEMAS-C 95098 (2001). Foundation for Water Research. UK.

Ervine, D.A. \& Falvey, H.R. (1987). "Behavior of turbulent jets in the atmosphere and in plunge pools." Proceedings of the Institution of Civil Engineers, Part 2, Vol. 83, pp. 295-314.

Federspiel, M., Bollaert, E.F.R. and Schleiss, A. (2009). "Response of an intelligent block to symmetrical core jet impact." Proceedings of the 33rd Congress of IAHR, ISBN: 978-94-90365-01-1, Vancouver, Canada, 9.-14. August 2009, CD-Rom, 2009, pp. 3573-3580.

Manso, P., Bollaert, E.F.R. and Schleiss, A.J. (2006). "Impact pressures of turbulent high-velocity jets plunging in pools with flat bottom." Experiments in Fluids.

Mason, P.J. and Arumugam, K. (1985). "A Review of 20 Years of Scour Development at Kariba Dam." Int. conf. on the Hydraulics of Floods and Flood Control, Cambridge, England. 